\title{
A Study on The Minimum Exposure Dose For Image Formation In X-Ray Imaging of Extremity
}

\author{
Sinyoung Y $\mathbf{u}^{1}$ \\ ${ }^{1}$ Ph.D., Dept. of Radiological Science, Far East University, 76-32, Daehak-gil, Gamgok-myeon, Eumsung-gun, \\ Chungcheongbuk-do, 27601, Korea
}

Article History:Received:11 February 2021; Accepted: 27 March 2021; Published online: 5 April 2021

\begin{abstract}
Excellent performance of the digital detector can generate medical images at a lower dose. However, if a certain level of dose exposure of the sensor detector can generate an image to recognize it.

In this study, the exposure conditions of the forearm, femur, and ankle joint tests currently used in clinical trials were tested with lower doses. The resolution pattern was analyzed using the Image $\mathbf{J}$ program, and the results were obtained by analyzing the Interactive 3D Surface Plot. In addition, by measuring the surface dose and absorbed dose, the digital detector finds the minimum conditions to make an image, and also checks the effect of dose reduction.

The image of the obtained resolution pattern was analyzed by Plot profile using Image J program. It was confirmed that the gray value width decreased at the same pixel distance as the exposure conditions decreased. Even if the exposure conditions were lowered from step 1 to step 4, which is the standard condition of the forearm test, the resolution showed no difference at $1.4 \mathrm{Lp} / \mathrm{mm}$.

In the Interactive 3D Surface Plot, as the range of the gray scale lowered the exposure condition, the shape of the surface contours gradually became blurred and the contrast of black and white shades decreased. The resolution from step 1 to step 3 , which is the standard condition of the femur bone test, was $1.2 \mathrm{Lp} / \mathrm{mm}$, indicating no difference. Even if the exposure conditions were lowered from step 1 to step 4 , which is the standard condition for ankle examination, the resolution showed no difference at $1.4 \mathrm{Lp} / \mathrm{mm}$.

It is thought that it is necessary to accumulate a lot of data by further subdividing the stage of exposure conditions according to the thickness in more areas.
\end{abstract}

Keywords: Exposure dose; Absorbed dose; Surface dose; Interactive 3D surface plot; Plot profile.

\section{Introduction}

The digital X-ray imaging system has a wider dynamic range and dose linearity compared to the film-sensing system, which reduces the re-shooting rate and achieves a certain level of image quality by adjusting the gray scale. The dynamic range in the digital detector can be expressed by the ratio of the maximum and minimum values of the pixel, and is effective only when the absorbed dose of the detector and the pixel value are in a linear relationship (Huda W et al., 1997; Rong, X.J et al., 2000; Floyd, C.E et al., 2001). Owing to this advantage, excessive doses could be used without degrading the image quality, and in turn, a constant image quality could be maintained even at small doses. The use of medical radiation can be helpful as well as patient community. However, excessive use of radiation can be dangerous for patients and users. Therefore, appropriate protection and management of medical radiation has been proposed, and the principle of medical radiation shielding lies in the justification and optimization of behavior (M. Köner et al., 2007; M. Yaffe et al., 1997). If the benefit of the purpose of the examination can be obtained more than the damage to the patient from the radiation examination, the justification is determined by careful judgment (Kroft LJ et al.,2006). And optimization is to keep the exposure dose as low as possible while obtaining optimal results from radiographic examinations performed on patients. Living radiation examining a patient using a medical radiation requires a constant effort and research (Okka WH et al.,2005). As system use and management become more convenient, careful management and effort are required by the user, the Radiological Technologists. Replacing the existing film / screen system inspection with digital radiography, there have been remarkable advances in the management of patient dose and image quality of radiography (Rapp-Bernhardt, U et al.,2003). In chest radiography, the amorphous silicon flat plate detector showed a significant reduction in both effective dose and entrance skin dose (ESD) compared to film / screen system and CR system, and the image quality was also reduced. It appeared to be more improved than the field (Strotzer, M et al.,1998). In addition, in the skeletal system examination, the dose could be reduced without deterioration. In the study using the hand phantom, the image quality was superior to that of the film / screen system under the same exposure conditions, and the diagnostic value was not degraded. Showed a decrease in dose (Strotzer, M et al.,2000; Bergh, B et al.,2000).

\section{Materials and Methods}

Based on the average exposure conditions of the forearm, femur, and ankle joints used in clinical exposure conditions, the experiment was conducted by lowering the tube voltage and tube current step by step for the human phantom. Images are acquired for each condition, and surface and absorbed doses are measured. The X-

*Corresponding author: Sinyoung Yu

Ph.D., Dept. of Radiological Science, Far East University, 76-32, Daehak-gil, Gamgok-myeon, Eumsung-gun, Chungcheongbuk-do, 27601, Korea

Email : ryusinyoung@gmail.com 
ray lead bar phantom is photographed under each stage of exposure conditions to obtain an image. The acquired image is analyzed using image analysis program Image $\mathrm{j}$.

\section{Resolution pattern test}

An image is acquired by photographing the $\mathrm{X}$-ray lead bar phantom under exposure conditions at each stage. The resolution of the X-ray lead bar phantom to the visible section was measured as shown in Figure 1.
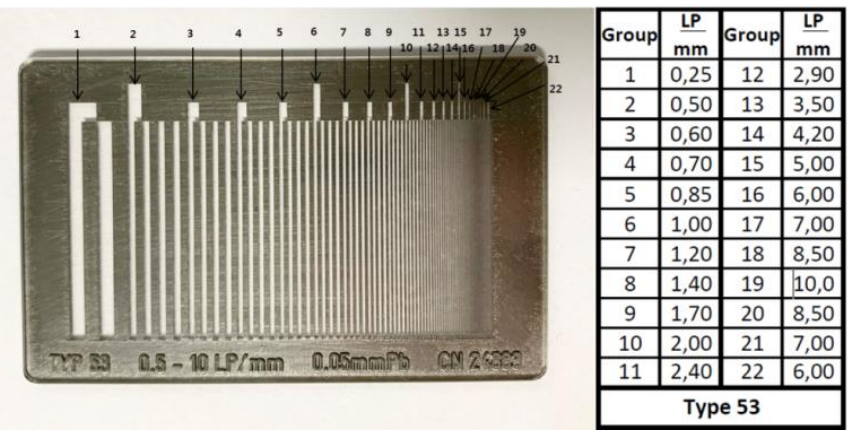

Figure 1. Hüttner standard X-ray lead bar phantom TYP 5

The acquired image is analyzed to as profile plot as shown in Figure 2 using an image analysis program, the image j.

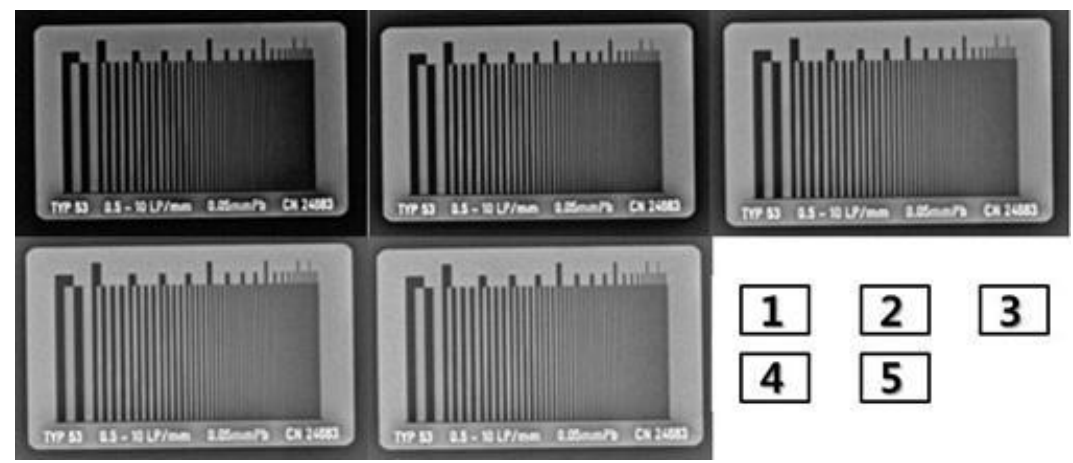

Figure 2. X-ray lead bar phantom image exposed at each stage condition

\section{Interactive 3D surface plot}

Interactive 3D surface plot analysis is performed on the images acquired using the image analysis program Image $\mathrm{j}$, as shown in Figure 3.

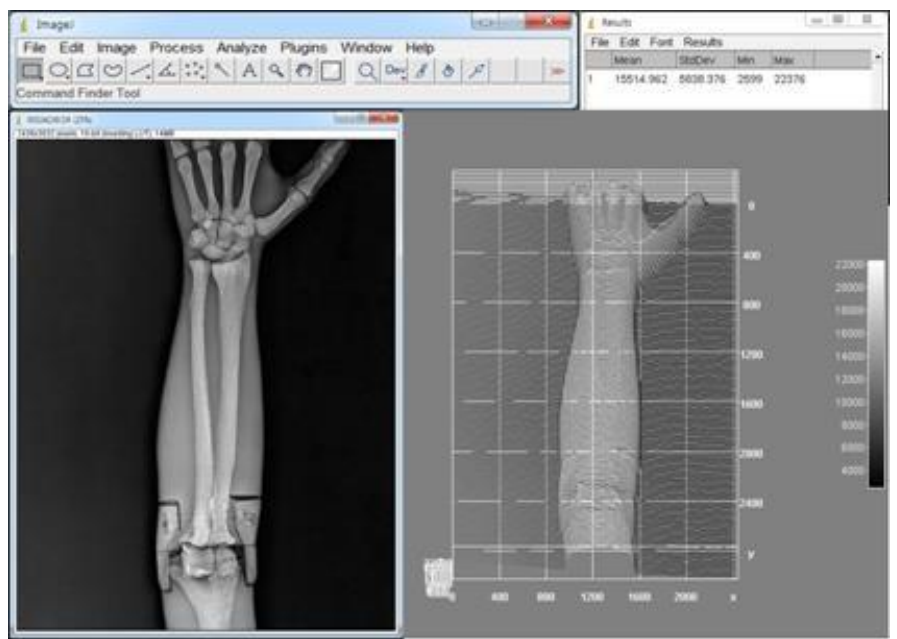

Figure 3. Interactive 3D surface plot analysis using Image $\mathbf{J}$

\section{Absorbed dose and Surface dose}

Forearm, femur, and ankle joints, surface dose and absorbed dose are measured using a human phantom for 
each condition. Measurements were performed using the Unifors RaySafe Xi Semiconductor dosimeter shown in Figure 4.

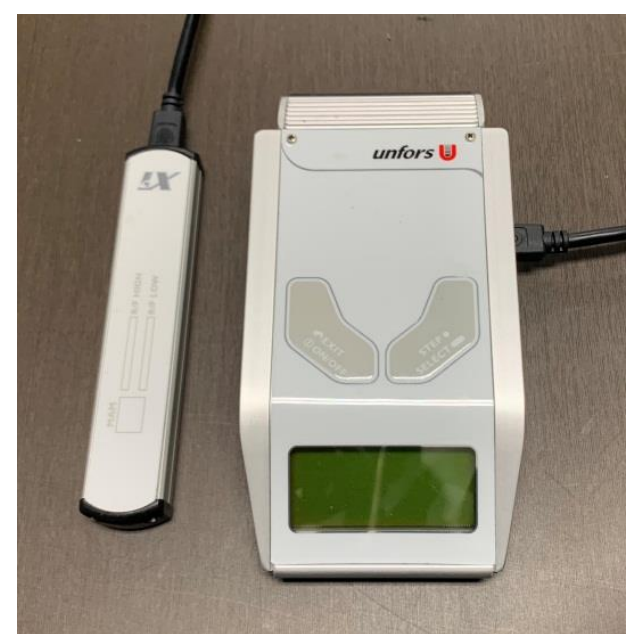

Figure 4. Unifors RaySafe Xi Semiconductor dosimeter

\section{Results and Discussion}

The image of the obtained resolution pattern was analyzed by Plot profile using Image $\mathrm{J}$ program. It was confirmed that the gray value width decreased at the same pixel distance as the exposure conditions decreased as showns in Figure 5.

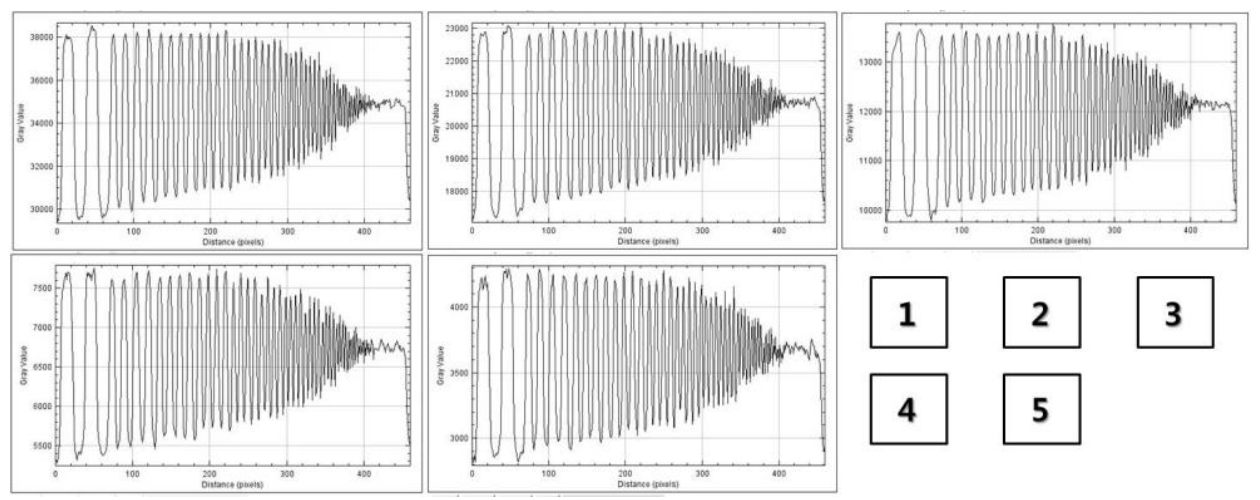

Figure 5. Plot profile in Resolution pattern

In the forearm test conditions step 1, step 2, step 3, and step 4, the resolution was $1.4 \mathrm{Lp} / \mathrm{mm}$ and in step 5 it was $1.2 \mathrm{Lp} / \mathrm{mm}$. Under the forearm test exposure condition, step 5, the absorbed dose decreased by $73.38 \%$ compared to step 1. Surface dose was reduced by $71.99 \%$ compared to step 1 in exposure condition step 5 as showns in Table 1.

Table 1. The absorbed dose ( $\mathrm{mGy}$ ) and the surface dose $(\mathrm{mR})$ in the Forearm exposure conditions

\begin{tabular}{c|c|c|c}
\hline Step & Lp / mm & mGy & $\mathrm{mR}$ \\
\hline 1 & 1.4 & 0.139 & 35.06 \\
\hline 2 & 1.4 & 0.101 & 25.90 \\
\hline 3 & 1.4 & 0.072 & 18.74 \\
\hline 4 & 1.4 & 0.052 & 13.61 \\
\hline
\end{tabular}




\begin{tabular}{l|l|l|l}
\hline 5 & 1.2 & 0.037 & 9.82 \\
\hline
\end{tabular}

In the femur test conditions step 1, step 2, step 3, the resolution was $1.4 \mathrm{Lp} / \mathrm{mm}$ and in step4 and step 5 it was $1.2 \mathrm{Lp} / \mathrm{mm}$. Under the femur test exposure condition, step 4, the absorbed dose decreased by $68.63 \%$ compared to step 1 . Surface dose was reduced by $65.15 \%$ compared to step 1 in exposure condition step 4 as showns in Table 2.

Table 2. The absorbed dose (mGy) and the surface dose $(\mathrm{mR})$ in the Femur exposure conditions

\begin{tabular}{c|c|c|c}
\hline Step & Lp / mm & mGy & mR \\
\hline 1 & 1.2 & 0.220 & 82.90 \\
\hline 2 & 1.2 & 0.153 & 59.65 \\
\hline 3 & 1.2 & 0.103 & 41.44 \\
\hline 4 & 1.0 & 0.069 & 28.89 \\
\hline 5 & 1.0 & 0.046 & 20.06 \\
\hline
\end{tabular}

In the ankle joint test conditions step 1, step 2, step 3, and step 4, the resolution was $1.4 \mathrm{Lp} / \mathrm{mm}$ and in step 5 it was $1.2 \mathrm{Lp} / \mathrm{mm}$. Under the forearm test exposure condition, step 5, the absorbed dose decreased by $84.52 \%$ compared to step 1. Surface dose was reduced by $81.19 \%$ compared to step 1 in exposure condition step 5 as showns in Table 3.

Table 3. The absorbed dose (mGy) and the surface dose $(\mathrm{mR})$ in the Ankle joint exposure conditions

\begin{tabular}{c|c|c|c}
\hline Step & Lp / mm & mGy & $\mathrm{mR}$ \\
\hline 1 & 1.4 & 0.084 & 20.84 \\
\hline 2 & 1.4 & 0.056 & 14.05 \\
\hline 3 & 1.4 & 0.036 & 9.50 \\
\hline 4 & 1.4 & 0.023 & 6.29 \\
\hline 5 & 1.2 & 0.013 & 3.92 \\
\hline
\end{tabular}

As a result of the analysis of the interactive 3D surface plot of the Forearm phantom image, it was confirmed that the shape of the surface contour is gradually blurred and the contrast of black and white shades decreases as showns in Figure 6. 


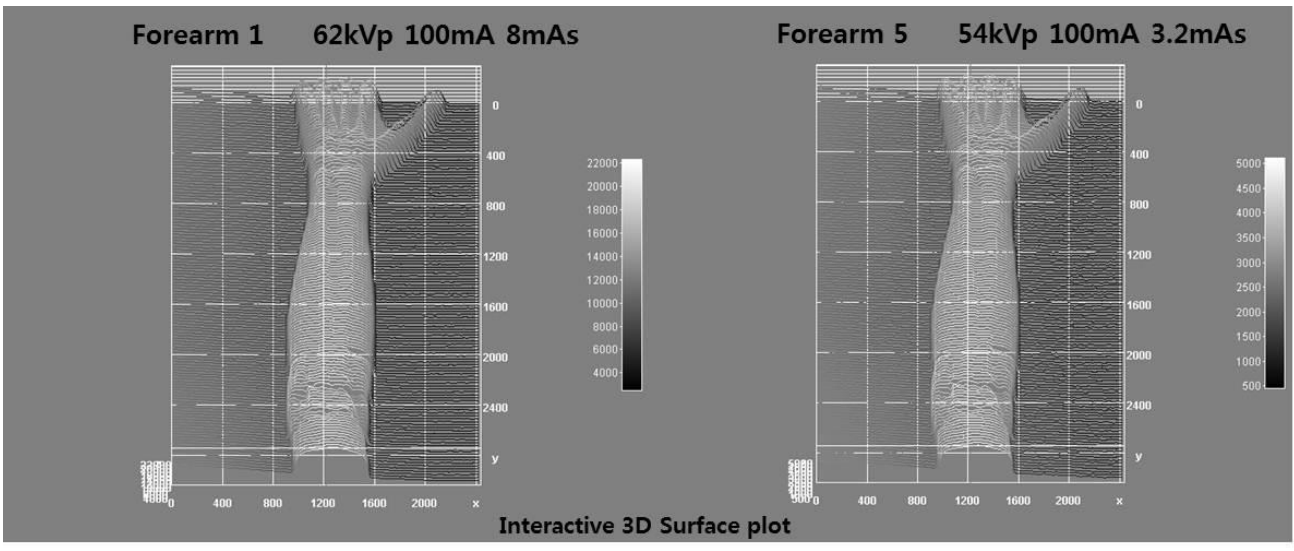

Figure 6. Comparative analysis of interactive 3D Surface Plot between Forearm step 1 condition and step 5 condition

As a result of the analysis of the interactive 3D surface plot of the Femur phantom image, it was confirmed that the shape of the surface contour is gradually blurred and the contrast of black and white shades decreases as showns in Figure 7.

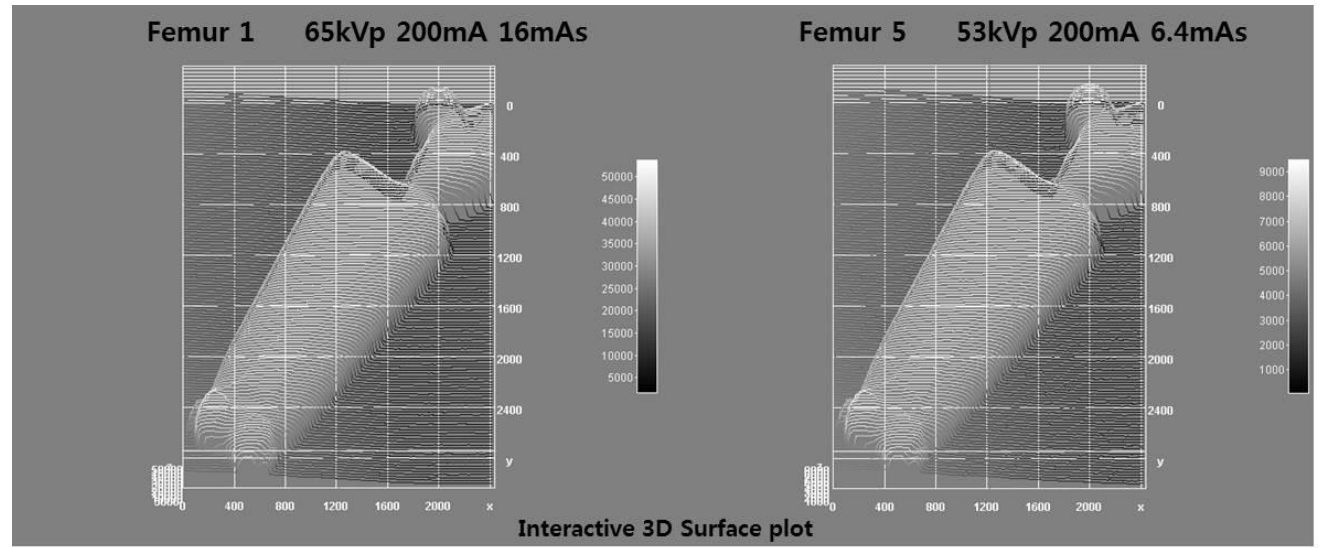

Figure 7. Comparative analysis of interactive 3D Surface Plot between Femur step 1 condition and step 5 condition

As a result of the analysis of the interactive 3D surface plot of the Ankle joit phantom image, it was confirmed that the shape of the surface contour is gradually blurred and the contrast of black and white shades decreases as showns in Figure 8.

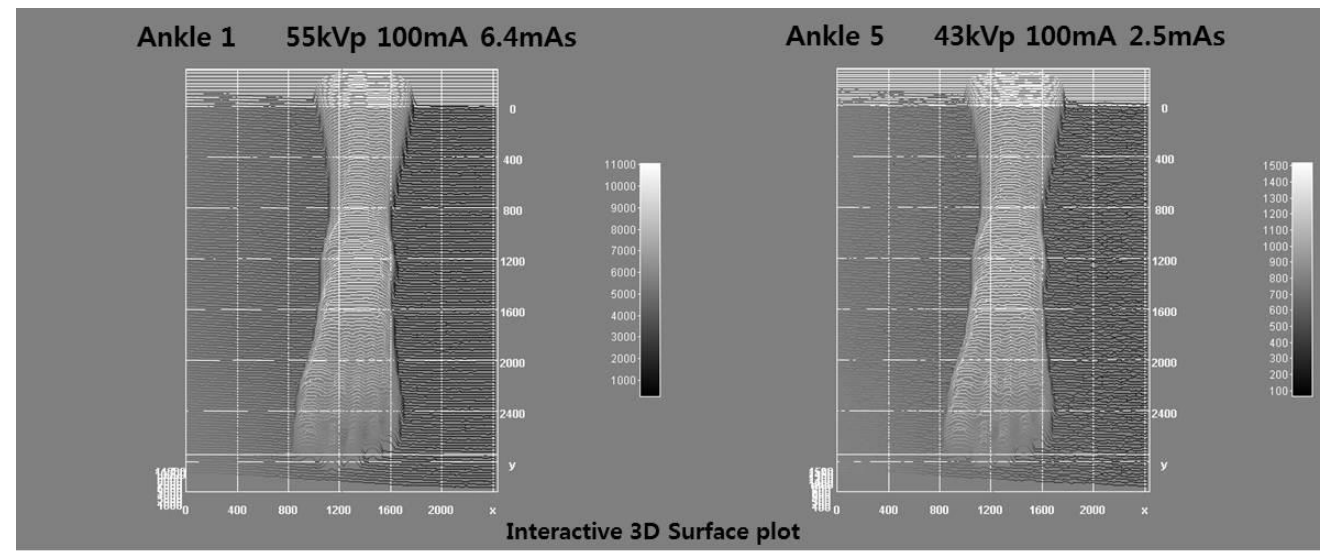

Figure 8. Comparative analysis of interactive 3D Surface Plot between Ankle step 1 condition and step 5 condition 
In this study, in order to find the minimum dose for which an image can be established, the exposure conditions currently used in clinical trials were examined step by step and the original data were analyzed in various ways. In the resolution, the spatial frequency was $1.0 \sim 1.4 \mathrm{Lp} / \mathrm{mm}$, and this value showed about 1.5 times higher resolution than the film image.

As a result of analyzing the Interactive 3D Surface Plot, it was found that as the exposure conditions decreased, the range of the gray scale decreased and the shape of the surface contour gradually disappeared, resulting in inferior contrast.

As a result of visual evaluation, it was found that the Forearm test should use the exposure conditions of step 4 at least $56 \mathrm{kVp}, 100 \mathrm{~mA}, 4 \mathrm{mAs}$. It was confirmed that the absorbed dose was reduced by $62.58 \%$ and the surface dose was reduced by $61.18 \%$ compared to the step 1 condition. In the Femur test, it was found that the exposure conditions of step 3 should be over $59 \mathrm{kVp}, 200 \mathrm{~mA}$, and $10 \mathrm{mAs}$. It was confirmed that the absorbed dose decreased by $53.18 \%$ and the surface dose decreased by $50.01 \%$ compared to the step 1 condition. In the ankle joint test conditions, it was found that the exposure conditions of step 3, $49 \mathrm{kVp}, 100 \mathrm{~mA}$, and $4 \mathrm{mAs}$, should be used. It was confirmed that the absorbed dose was reduced by $57.14 \%$ and the surface dose was reduced by $54.41 \%$ compared to the step 1 condition.

\section{Conclusion}

This study was conducted using a human model phantom made of tissue equivalent material. forearm, femur, and ankle joint did not have any problems in conducting the experiment and analyzing the images, but it is expected to have difficulty in accurate image evaluation because internal organs or spaces cannot be identified in the head or abdomen examination. In addition, due to the constant thickness of the human body model phantom, it was unfortunate that the results could not be obtained in various body types that could be blown by real patients. In this study, X-rays were exposed by dividing the condition into 5 stages in a total of 3 regions, and it is thought that the stages of the exposure conditions should be further subdivided according to the thickness in more regions to accumulate a lot of data. If these studies continue, you can expect to develop technology for digital detector sensors based on them.

Funding Support - The authors declare that they have no funding support for this study.

Conflict of Interest - The authors declare that they have no conflict of interest.

\section{References}

1. Bergh, B., Pietsch, M. and Vogl, T.J. , 2000 , Internet technology for clinical applications in a digital radiography department, Radiologe 40, pp.371-387

2. Floyd, C.E., Warp, R.J., Dobbins III, J.T., Chotas, H.G., Baydush, A.H., Vargas-Voracek, R. and Ravin C.E. , 2001, Imaging characteristics of an amorphous silicon flat-panel detector for digital chest radiography, Radiology 218, pp.683-688

3. Huda, W., Rill, L.N. and Bruner, A.P. , 1997, computed radiography phosphors and screen-film systems, Relative speeds of Kodak - 85 -. Med. Phys. 24, pp.1621-1628.

4. Kroft LJ, Veldkamp WJ, Mertens BJ, van Delft JP, Geleijns J. , 2006, Detection of simulated nodules on clinical radiographs: dose reduction at digital posteroanterior chest radiology. , Radiology 241, pp.3 92-398

5. M. Köner, C. H. Weber, S. Wirth, K.-J. Pfeifer, M. F. Reiser, and M. Treitl, 2007, “Advances in Digita 1 Radiography: Physical Principles and System Overview1", Radiographics, vol.27, pp.675-686.

6. M. Yaffe and J. Rowlands, 1997, "X-ray detectors for digital radiography", Physics in Medicine and Bi ology, 42, pp.1.

7. Okka WH, Claude BS, Michael S, et al. , 2005 , Chest radiography with a flat-panel detector; Image qu ality with dose rediction after copper filtration., Radiology 237, pp.691-700

8. Rapp-Bernhardt, U., Roehl, F.W., Gibbs, R.C, Schmidl, H. and Bernhardt, T.M. , 2003 , Flat-Panel Xray Detector Based on Amorphous Silicon Versus Asymmetric Screen-Film System: Phantom Study of Dose Reduction and Depiction of Simulated Findings, Radiology, 227, pp.484-492

9. Rong, X.J., Shaw, C.C., Liu, X., Lemacks, M.R. and Thompson, S.K. , 2000 , Comparison of an amorphous silicon/cesium iodide flat-panel digital chest radiography system with screen/film and computed radiography systems: a contrast-detail phantom study, - 89 - Med Phys 28, pp. 2328-2335

10. Strotzer, M., Gmeinwieser, J., Volk, M., Frund, R., Seitz, J., Albrich, H. and Feuerbach, S. , 1998 , Clinical application of a flat-panel X-ray detector based on amorphous silicon technology: image quality and potential for radiation dose reduction in skeletal radiography, AJR Am J Roentgenol, - 91 -171 , pp.23-27

11. Strotzer, M., Volk, M., Wild, T., Landenberg, P. von. and Feuerbach, S. , 2000 , Simulated Bone Erosions in a Hand Phantom: Detection with Conventional Screen-Film Technology versus Cesium Iodide-Amorphous Silicon Flat-Panel Detector, Radiology, 215, pp.512-515 